\title{
SCANNING THE SONNEBERG PLATE ARCHIVE WITH DIA
}

\author{
P. KROLL ${ }^{1}$ and P. NEUGEBAUER ${ }^{2}$ \\ ${ }^{1}$ Sonneberg Observatory, D-96515 Sonneberg, Germany \\ 2 Lehr- und Forschungsbereich Theoretische Astrophysik Tübingen, Auf der \\ Morgenstelle 10, D-72074 Tübingen, Germany
}

\section{Introduction}

DIA (Digital Image Analyzer) is a new dedicated and low cost CCD line scanner, which has been invented at Sonneberg Observatory in collaboration with the Institute for Theoretical Astrophysics at Tubingen for scanning selected fields of the Sonneberg Plate Archive.

\section{Equipment}

1) Scanner camera:

- OPTOSCAN CCD line camera

- CCD line with 5000 pixels, 7200 steps

- data depth: 12 bit

- integration time: 7 ... $64 \mathrm{~ms}$

- optics: Rodenstock Rodagon-WA 1:4, $f=60 \mathrm{~mm}$

- interface: SCSI-2

2) Base:

- adapted commercial scanner stand for astrographic applications with height compensated adjustable camera in a dust-free environment

3) Moving table:

- total accessible size: $20 \mathrm{~cm} \times 20 \mathrm{~cm}$

- 2 stepping motors: $X$ and $Y$ (rotation optional)

- positioning accuracy: $1.7 \mu \mathrm{m}$

4) Computer:

- IRIS Indigo Workstation 3000 of Silicon Graphics Inc.

- also possible: Sun Sparcstations, VAXstations of DEC with integrated SCSI-controller

5) Software:

- POLYSCAN of SCIENCE \& COMPUTING GmbH Tübingen

- operated via an OSF/Motif-based graphical user interface 
- scanned pictures stored in standard TIFF (=Tag Image File Format)

- usage in interactive mode as well as batch mode

6) Backup medium:

- Exabyte Cassette Drive with 5 GB per tape

- moderately fast, reliable and inexpensive (less than \$5/GB)

7) Distributor and Costs:

- SCIENCE \& COMPUTING GmbH, Tubingen, Germany

- purchase price of whole device (scanner, base, moving table, workstation): less than $\$ 80,000$

\section{Scanning of Sonneberg Plates}

- Scanning schedule in interactive mode:

1) focusing and tuning the resolution

2) choosing the optimal dynamic range (varying the objective's diaphragm and/or integration time

3) choosing the region to be scanned

4) scanning process ( 3 minutes, $36 \mathrm{MB}$ of data).

- Scanning schedule in batch mode:

1) focusing and tuning the resolution

2) starting the AUTOSCAN program for scanning the whole plate: ca. 1 hour for a sky patrol plate of size $12 \mathrm{~cm} \times 12 \mathrm{~cm}$ (resolution 10 $\mu \mathrm{m}$, ca. $150 \mathrm{MB}$ ), ca. 5 to 6 hours for a field patrol plate of size $30 \mathrm{~cm} \times 30 \mathrm{~cm}$ (ca. $1 \mathrm{~GB}$ )

3) writing the data from harddisk to Exabyte drive (ca. 1 hour/GB).

\section{Brightness Determination}

For details see: Kroll, P. \& Neugebauer, P., 1993. Astron. \& Astrophys., 273, 341.

Application to astrometry has not yet been tested. 